\title{
Polarized proton acceleration in ultraintense laser interaction with near-critical-density plasmas
}

\author{
X. F. Li ${ }^{1,2,3}$ P. Gibbon $\odot,,^{1,4, *}$ A. Hützen $\odot,,^{5,6}$ M. Büscher $\odot,{ }^{5,6}$ S. M. Weng $\odot,{ }^{2,3, \dagger}$ M. Chen $\odot,{ }^{2,3}$ and Z. M. Sheng $\oplus^{2,3,7,8}$ \\ ${ }^{1}$ Institute for Advanced Simulation, Jülich Supercomputing Centre, Forschungszentrum Jülich, 52425 Jülich, Germany \\ ${ }^{2}$ Key Laboratory for Laser Plasmas (Ministry of Education), School of Physics and Astronomy, \\ Shanghai Jiao Tong University, Shanghai 200240, China \\ ${ }^{3}$ Collaborative Innovation Center of IFSA, Shanghai Jiao Tong University, Shanghai 200240, China \\ ${ }^{4}$ Centre for Mathematical Plasma Astrophysics, Katholieke Universiteit Leuven, 3000 Leuven, Belgium \\ ${ }^{5}$ Peter Grünberg Institut (PGI-6), Forschungszentrum Jülich, 52425 Jülich, Germany \\ ${ }^{6}$ Institut für Laser- und Plasmaphysik, Heinrich-Heine-Universität Düsseldorf, 40225 Düsseldorf, Germany \\ ${ }^{7}$ SUPA, Department of Physics, University of Strathclyde, Glasgow G4 ONG, United Kingdom \\ ${ }^{8}$ Tsung-Dao Lee Institute, Shanghai Jiao Tong University, Shanghai 200240, China
}

(Received 25 March 2021; accepted 8 July 2021; published 30 July 2021)

\begin{abstract}
The production of polarized proton beams with multi-GeV energies in ultraintense laser interaction with targets is studied with three-dimensional particle-in-cell simulations. A near-critical density plasma target with prepolarized proton and tritium ions is considered for the proton acceleration. The prepolarized protons are initially accelerated by laser radiation pressure before injection and further acceleration in a bubblelike wakefield. The temporal dynamics of proton polarization is tracked via the Thomas-Bargmann-Michel-Telegdi equation and it is found that the proton polarization state can be altered by both the laser field and the magnetic component of the wakefield. The dependence of the proton acceleration and polarization on the ratio of the ion species is determined and it is found that the protons can be efficiently accelerated as long as their relative fraction is less than $20 \%$, in which case the bubble size is large enough for the protons to obtain sufficient energy to overcome the bubble injection threshold.
\end{abstract}

DOI: 10.1103/PhysRevE.104.015216

\section{INTRODUCTION}

With the development of laser technology, especially chirped pulse amplification [1], remarkable progress has been achieved in the field of laser-plasma acceleration [2,3]. Long after laser-driven wakefield acceleration (LWFA) was first proposed by Tajima and Dawson [4], electron beams with quasimonoenergetic peaks up to $7.8 \mathrm{GeV}$ were generated using a peak laser power of $850 \mathrm{TW}$ interacting with a $20-\mathrm{cm}$ capillary charge waveguide in [5]. The maximum energy for laser-driven ion acceleration is around $85 \mathrm{MeV}$, via a high-energy laser incident on micrometer-thick plastic targets [6]. Protons near $100 \mathrm{MeV}$ have been obtained through a hybrid scheme of radiation pressure and sheath acceleration [7]. These plasma-based accelerators have prompted a new class of diagnostic techniques different from those common to traditional accelerators [8]. In order to effectively utilize and develop laser plasma acceleration, many characteristics of particle beams, e.g., energy spread, charge, pulse duration, and emittance, have been steadily improved. However, the polarization of particles has rarely been studied in plasma-based acceleration [9].

Polarization is defined as the collective spin state of a particle beam. It is commonly employed in nuclear physics [10], high-energy physics [11], and material physics [12]. For

\footnotetext{
*p.gibbon@fz-juelich.de

†wengsuming@gmail.com
}

laser-driven accelerators, there are two approaches leading to polarized particle beams: a de novo polarization buildup from an unpolarized target in the interaction with laser and polarization preservation of prealigned spins during the acceleration. In the first instance, the spin polarization of an ultrarelativistic electron beam has been investigated through colliding with an intense laser pulse in the quantum radiationdominated regime [13]. A possible way to produce highly polarized positron beams was also proposed via interaction of an ultrarelativistic electron beam with counterpropagating two-color intense laser pulses [14]. For a prepolarized target, it has been demonstrated recently that nuclear and electron spin-polarized $\mathrm{H}$ and $\mathrm{D}$ densities of at least $10^{19} \mathrm{~cm}^{-3}$ with 10-ns lifetimes can be produced by photodissociation of $\mathrm{HBr}$ and DI with circularly polarized UV light pulses [15]. Using this kind of prepolarized target, Wen et al. demonstrated that kiloampere polarized electron beams can be produced through LWFA in the bubble regime [16]. Furthermore, a vortex laser interacting with prepolarized plasma was proposed to produce energetic electrons with high polarization and beam charge [17].

Because the proton is 1836 times heavier than the electron or positron, it is much more difficult to align the spins of protons via laser-solid interaction, as demonstrated by Büscher and co-workers [18-20]. For this reason, acceleration of polarized proton beams has been investigated usually using a prepolarized target composed of two different ion species close to the critical density for a Ti:sapphire laser. By irradiating prepolarized monatomic gases from 
photodissociated hydrogen halide molecules with a petawatt laser, proton beams with nearly $100 \mathrm{MeV}$ energy and $80 \%$ polarization via magnetic vortex acceleration mechanism [21] were predicted. In order to obtain several-GeV protons from near-critical plasma targets, wakefield acceleration offers a good option. The work of Shen et al. showed that the protons can be trapped and accelerated efficiently in the bubble regime, where the plasma consists of a small proportion of protons and a large proportion of heavier tritium ions [22]. It should be noted that the density ratio of protons and heavier ions is different in the two above studies. In addition, the protons need to be preaccelerated to a sufficient energy in order to be injected into the wakefield. In the work of Liu et al. it was shown that protons preaccelerated by the radiation pressure acceleration in a thin solid foil could be captured by the LWFA in an underdense gas [23].

In this paper, preacceleration of protons via direct laser acceleration with an ultraintense circularly polarized laser is proposed. This initial stage is followed by proton acceleration in a wakefield, where the polarization dynamics is also examined in detail. Finally, the influence of the proton-to-ion ratio on the acceleration and polarization is investigated.

\section{SIMULATION METHOD}

A series of simulations was performed to study the polarization dynamics during the acceleration of prepolarized protons with a modified version of the three-dimensional particle-in-cell (PIC) code EPOCH [24]. A laser pulse with $\lambda=$ $0.8 \mu \mathrm{m}$ wavelength and clockwise polarization is propagated in the $x$ direction with a focused transverse Gaussian profile. The spot size is $w_{0}=10 \lambda$ and the pulse duration is $\tau=$ $20 \mathrm{fs}$. The normalized laser amplitude is $a_{0}=e E_{0} / m_{e} \omega c=$ $316 / \sqrt{2}$, corresponding to a laser intensity $I_{0}=2.14 \times 10^{23}$ $\mathrm{W} / \mathrm{cm}^{2}$, as adopted in Ref. [22], where $e$ and $m_{e}$ are the electron mass and charge, respectively. The simulation mesh resolution is $d x=0.03125 \lambda$ and $d y=d z=0.5 \lambda$ in the longitudinal and transverse directions, respectively. There are four macroparticles per species per cell. The moving window simulation box is $50 \lambda \times 90 \lambda \times 90 \lambda$ with open boundary conditions in each direction. The proton polarization is defined by its spin vector $\mathbf{s}$, which has an absolute value of 1 and a direction calculated from the Thomas-Bargmann-MichelTelegdi (TBMT) equation $d \mathbf{s} / d t=-\mathbf{\Omega} \times \mathbf{s}[25,26]$,

$$
\begin{aligned}
\mathbf{\Omega}=\frac{q}{m c} & {\left[\left(a_{p}+\frac{1}{\gamma}\right) \mathbf{B}-\left(\frac{a_{p} \gamma}{\gamma+1}\right)\left(\frac{\mathbf{v}}{c} \cdot \mathbf{B}\right) \frac{\mathbf{v}}{c}\right.} \\
& \left.-\left(a_{p}+\frac{1}{1+\gamma}\right) \frac{\mathbf{v}}{c} \times \mathbf{E}\right],
\end{aligned}
$$

where $m$ and $q$ are the proton mass and charge, respectively, $a_{p}=0.5\left(g_{p}-2\right) \simeq 1.79$ is the dimensionless anomalous magnetic moment of the proton, $\gamma$ is the Lorentz factor of the proton velocity, $c$ is the light speed in vacuum, $\mathbf{B}$ is the magnetic field, and $\mathbf{E}$ is the electric field in the laboratory frame. Equation (1) can be evaluated using an adapted version of the standard Boris operator splitting method commonly used for the momentum integration in PIC codes [27,28]. Although the particle spin can in principle be altered by the Stern-Gerlach and Sokolov-Ternov effects, these have been neglected in

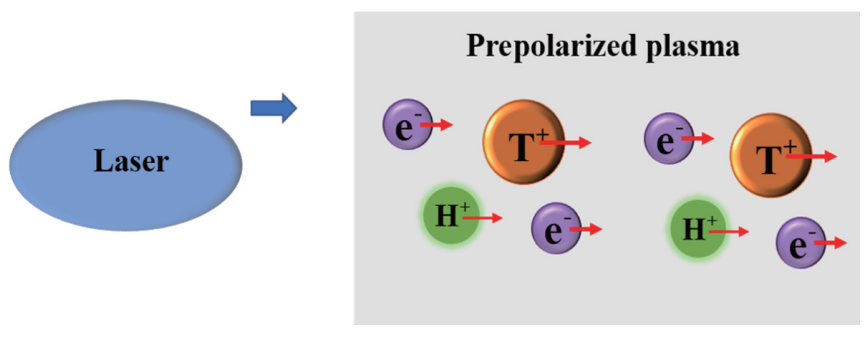

FIG. 1. Schematic of laser prepolarized plasma interaction. The polarization direction of particles is denoted by red arrows and is aligned with the propagation direction of the laser. The plasma is composed of electrons $\left(e^{-}\right)$, protons $\left(H^{+}\right)$, and tritium ions $\left(T^{+}\right)$.

our study based on the prior work of Ref. [29]. The simulation geometry is shown in Fig. 1, where the initial electron, proton, and tritium densities are $1.5 \times 10^{21}, 1.0 \times 10^{20}$, and $1.4 \times 10^{21} \mathrm{~cm}^{-3}$, respectively. The plasma density is uniform and the vacuum length at the left edge is $15 \lambda$. The proton-totritium density ratio is $1: 14$. The target is initially prepolarized in the $x$ direction [16], as depicted by the red arrows in Fig. 1.

\section{Benchmarking the TBMT equation}

The TBMT equation was previously analyzed in detail in Ref. [25], which discussed two analytical solutions: the Wien filter and uniform magnetic field. The Wien filter case is a uniform magnetic field plus an orthogonal electric field such that the net Lorentz force vanishes: $\mathbf{E}+\boldsymbol{\beta} \times \mathbf{B}=0$. The velocity $\boldsymbol{\beta}$ is orthogonal to both the electric and magnetic fields. Here the acceleration of the particle $\dot{\boldsymbol{\beta}}=0$ and the spin precession vector is given by

$$
\boldsymbol{\Omega}=\frac{g_{p} q}{m c}(\mathbf{B}-\boldsymbol{\beta} \times \mathbf{E})=\frac{g_{p} q}{\gamma^{2} m c} \mathbf{B} .
$$

The particle moves in a straight line, but the spin precesses around the magnetic field. In the case of a uniform vertical magnetic field with no electric field, the gyrofrequency for horizontal circular motion is

$$
\omega_{\mathrm{rev}}=\frac{q}{\gamma m c} \mathbf{B},
$$

whereas the spin precession frequency is

$$
\boldsymbol{\Omega}=\frac{q}{m c}\left(a_{p}+\frac{1}{\gamma}\right) \mathbf{B} .
$$

The EPOCH particle integrator including the TBMT equation was verified using the two above cases. To do that, a bunch of protons with uniform energy $\gamma$ and density $n_{p}=$ $1.74 \times 10^{15} \mathrm{~cm}^{-3}$ was used, where the electromagnetic field generated by the proton beam can be ignored so that all the protons followed similar trajectories. Periodic boundaries were used and the grid resolution was $d x=0.02 \lambda$ and $d y=d z=0.05 \lambda$, where $\lambda=0.8 \mu \mathrm{m}$.

In the case of the Wien filter, the magnetic field $\mathbf{B}=B_{z} \mathbf{k}$ and the electric field $\mathbf{E}=E_{y} \mathbf{j}=\beta_{x} B_{z} \mathbf{j}$, where the proton propagates in the $x$ direction with $\boldsymbol{\beta}=\beta_{x} \mathbf{i}$, and $\mathbf{i}, \mathbf{j}$, and $\mathbf{k}$ are the unit vectors in the $x, y$, and $z$ directions, respectively. In this case, the proton orbit is a line in the $x$ direction, while the proton spin rotates in the $X Y$ plane with Eq. (2). When 

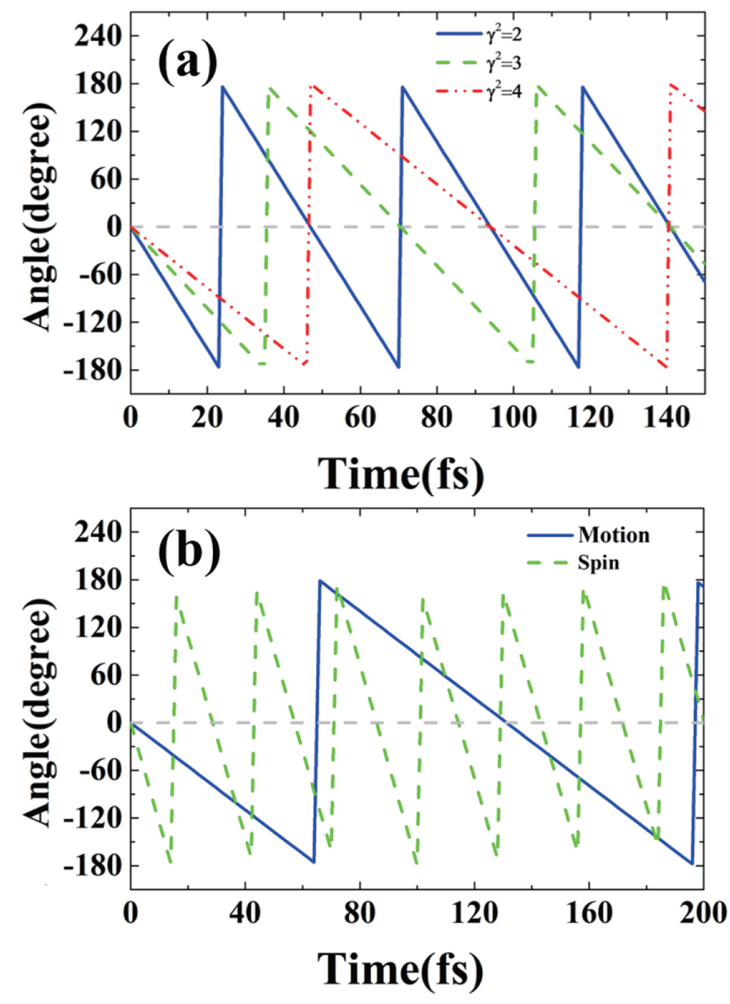

FIG. 2. Benchmark results for (a) the Wien filter case showing the evolution of angle of spin rotation for protons of different energies $\gamma^{2}=2$ (blue solid line), $\gamma^{2}=3$ (green dashed line), and $\gamma^{2}=4$ (red dotted line) and (b) the uniform vertical magnetic field case with $B_{z}=10^{6} \mathrm{~T}$, showing the history of the angle of orbit (blue solid line) and spin (green dashed line) rotation for protons with $\gamma=2$. The angles of spin and motion rotation are obtained from $\phi=\tan ^{-1}\left(s_{y} / s_{x}\right)$ and $\theta=\tan ^{-1}\left(p_{y} / p_{x}\right)$. The initial spin and velocity were both in the $x$ direction.

$B_{z}=10^{6} \mathrm{~T}$, the proton spin rotation period is

$$
T_{s}=2 \pi / \Omega \simeq 23.5 \gamma^{2}
$$

(in femtoseconds), in agreement with the numerical time histories of $\phi=\tan ^{-1}\left(s_{y} / s_{x}\right)$ in Fig. 2(a). In the case of uniform magnetic field $\mathbf{B}=B_{z} \mathbf{k}$, the proton orbit is a circle in the $X Y$ plane with frequency given by Eq. (3) and the proton spin also located at the $X Y$ plane according to Eq. (4). When $B_{z}=10^{6} \mathrm{~T}$ and $\gamma=2$, the periods of motion and spin are $T_{\text {rev }}=131$ fs and $T_{s}=28.62$ fs respectively, also borne out by the simulation results in Fig. 2(b).

\section{RESULTS AND DISCUSSION}

An intense laser pulse injected into an underdense plasma can result in a blowout state in which the electrons are expelled by the laser while the ions remain immobile. Owing to the charge separation field, the electrons return to the axis forming a positively charged bubble, which has a phase velocity equal to the group velocity of the driver laser. Increasing the plasma density to near-critical density results in a lower bubble velocity and an enhancement of the electromagnetic field in the bubble. Because of its longer exposure to a more intense accelerating field, the motion of protons cannot be ignored and some protons may be captured by the wakefield.

\section{A. Proton trapping and acceleration}

As shown in Fig. 3(a), the electron bubble is formed at $100 \mathrm{fs}$ and some electrons are injected into the tail of the bubble. There are also some protons distributed near the driving laser at the head of the bubble. The proton density forms a vortex microstructure. The rotation direction is the same as the laser polarization. For the laser amplitude $a_{0}=316 / \sqrt{2}$, the quiver velocity of protons would be $v_{p} / c=a_{0} / 1836=0.172$, which means that the protons will move significantly in the laser field. During the first stage of preacceleration, the proton motion is therefore directly affected by the ultraintense laser field.

This can be demonstrated by examining the dependence of the resultant kinetic energy distribution on the laser polarization in the first stage. Figures 3(b) and 3(c) show the distribution of proton density at same time with counterclockwise and linear polarization, respectively. Compared with the case of clockwise polarization, the vortex structure follows the laser polarization such that the microstructure is imprinted directly by the laser. When the laser is linearly polarized, the proton density takes on a multilayer form instead. These features are also reflected in the distribution of proton kinetic energy $E_{k}(x)$, as shown in Fig. 3(d) for the clockwise polarized laser. Here the proton energy has a periodic variation with the laser wavelength, another strong indication that the protons are directly modulated by the laser.

At later times, the protons with kinetic energy $E_{k}>4 \mathrm{GeV}$ are still located in the acceleration regime with $E_{x}>0$, as shown in Fig. 3(e). This means that the high-energy protons are able to catch up with the electron bubble and continue to gain energy in the wakefield. To track the acceleration dynamics further, nearly 2000 protons are selected with $E_{k}>4 \mathrm{GeV}$ at $400 \mathrm{fs}$. Their positions at $100 \mathrm{fs}$ are shown as white dots in Fig. 3(d); their subsequent positions relative to the longitudinal electric field profile at later times are displayed in Fig. 3(f). At $200 \mathrm{fs}$, these protons (orange dots) are still located within the accelerating region of the wakefield with $E_{x}>0$, although they undergo some slippage before regaining some ground between 200 and 400 fs. In other words, the protons are trapped in the wakefield at nearly $400 \mathrm{fs}$ and are subsequently accelerated continuously. On the other hand, their transverse position increases with time, implying that the bunch experiences a defocusing radial field, as shown in Fig. 5(d).

\section{B. Effect of laser polarization on energy gain}

A further series of simulations shows that the protons always slip out from the bubble regime and cannot be further accelerated if a linearly polarized (LP) laser with the same energy is used. For plasma waveguides, a circularly polarized (CP) laser is found to produce a higher electron temperature than a LP laser [30]. For electron acceleration in the laser-driven wakefield, it is found that using a $\mathrm{CP}$ laser can decrease the self-injection threshold in the nonpreformed plasma, compared to the case with a LP laser [31]. In a typical underdense plasma, the bubble size is nearly the same in the LP and CP cases. However, the situation is quite different 

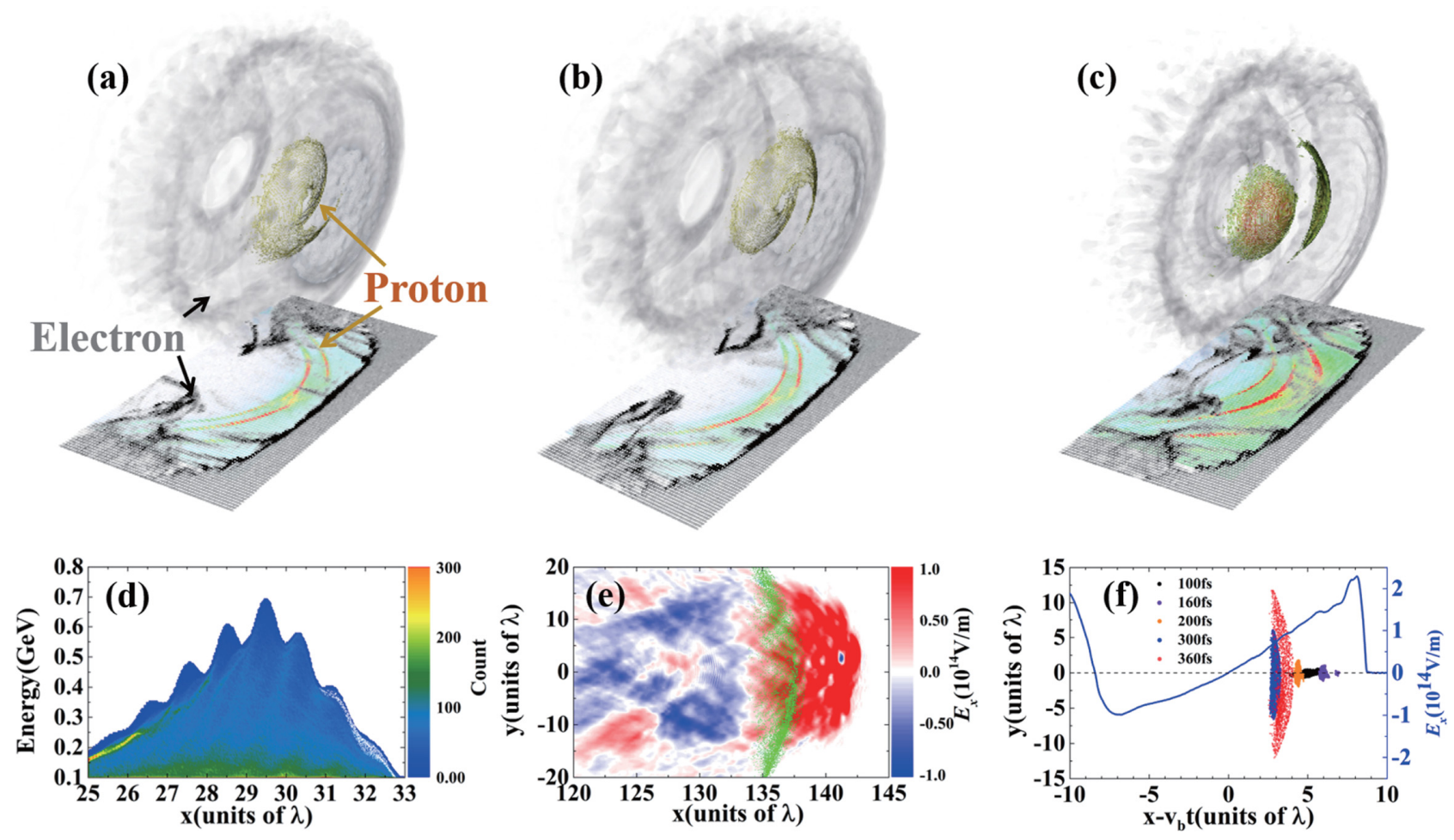

FIG. 3. Distribution of proton and electron densities at $100 \mathrm{fs}$ in cases with different laser polarization: (a) clockwise polarization, (b) counterclockwise polarization, and (c) linear polarization. (d) Spatial distribution of proton kinetic energy $E_{k}>0.1 \mathrm{GeV}$ along $x$ at $100 \mathrm{fs}$. (e) Distribution of $E_{x}$ at $400 \mathrm{fs}$. Protons with kinetic energy higher than $4 \mathrm{GeV}$ are denoted by green dots. (f) Position of selected protons [denoted by white dots in (d)] at different times in the bubble frame, where $v_{b}$ is the bubble velocity. The distribution of the longitudinal field on the axis at $120 \mathrm{fs}$ is shown as a blue solid line. The black dashed line shows the longitudinal field $E_{x}=0$. The laser intensity is $a_{0}=316 / \sqrt{2}$ and the initial electron density is $n_{e 0}=1.5 \times 10^{21} \mathrm{~cm}^{-3}$. The proton and tritium densities are $n_{p 0}=1.0 \times 10^{20} \mathrm{~cm}^{-3}$ and $n_{T 0}=1.4 \times 10^{21} \mathrm{~cm}^{-3}$, respectively.

for a near-critical-density plasma, in which it has been found that proton acceleration in the wakefield is more effective for a CP laser driver. For a LP laser pulse with the same energy, the longitudinal electric field $E_{x}(x)$ at $120 \mathrm{fs}$ is already obviously different from that produced by a CP laser, as shown in Fig. 4(a). The bubble size is smaller and the longitudinal field $E_{x}$ is weaker in the case of the LP laser. As a result, the protons gain higher energy with a CP laser, as shown in Fig. 4(b), enabling them to be injected into the wakefield. Another difference is shown in Fig. 4(c), which gives the energy phase space at 100 fs for the LP laser, clearly revealing a periodic structure at half the laser wavelength owning to the oscillating term of ponderomotive force [32].

\section{Proton spin dynamics and polarization preservation}

The original motivation of this study was to analyze the time evolution of the proton spin. The polarization of selected protons was investigated first. The distribution of the proton spin component $s_{x}$ at $100 \mathrm{fs}$ is plotted in Fig. 5(a). At this time, the $s_{x}$ values do not change significantly, remaining close to their initial value of $s_{x}=1$. Meanwhile, the spins exhibit a periodic structure at the laser wavelength. The corresponding proton positions and a map of $E_{y}\left(B_{z}\right)$ are shown in Fig. 5(c) [Fig. 5(e)]. The protons are located in the laser field near the axis, where the $E_{y}$ and $B_{z}$ of the wakefield could be ignored.
Here the laser electric field is approximately $10^{14} \mathrm{~V} / \mathrm{m}$, as shown in Fig. 5(c), with the corresponding magnetic field nearly $10^{6} \mathrm{~T}$. At $100 \mathrm{fs}$, the kinetic energy of the proton is not very large $\gamma \simeq 1$. From Eq. (1), $\Omega \simeq \frac{q}{m c}\left(a_{p}+\frac{1}{\gamma}\right) \mathbf{B}=$ $2.67 \times 10^{13} \mathrm{~Hz}$, where the cycle of rotation is nearly $23 \mathrm{fs}$, implying that the polarization of the proton beam could also be affected by the laser field directly. This means that the spin of the proton should also be considered, although there are many works focusing on the spin motion of electrons (or positrons) in ultraintense laser fields $[9,33,34]$.

By contrast, later on at $400 \mathrm{fs}$ [in Fig. 5(b)], the polarization distribution of $s_{x}$ ranges from -1 to 1 ; in particular, the spin goes negative for trailing protons. At this time, these protons have slipped backward and are mainly distributed in the field generated by the cavity wakefield. In the bubble regime as presented in Fig. 5(d), the transverse field usually focuses electrons, but defocuses for positively charged particles, for example, positrons and protons. The transverse size of a proton bunch will therefore increase in time. Here it can be seen that the transverse extent of the proton bunch has increased, especially its rear portion, as shown in Fig. 5(f). These are located at the sheath of the bubble, where $B_{z}$ is larger and the polarization rotates faster. Here the maximum $B_{z}$ is nearly $0.2 \times 10^{6} \mathrm{~T}$, so the rotation period is only $100 \mathrm{fs}$ for protons with $\gamma \simeq 1$. For trapped protons located near the laser axis, this period will be longer, resulting in less spin rotation. 

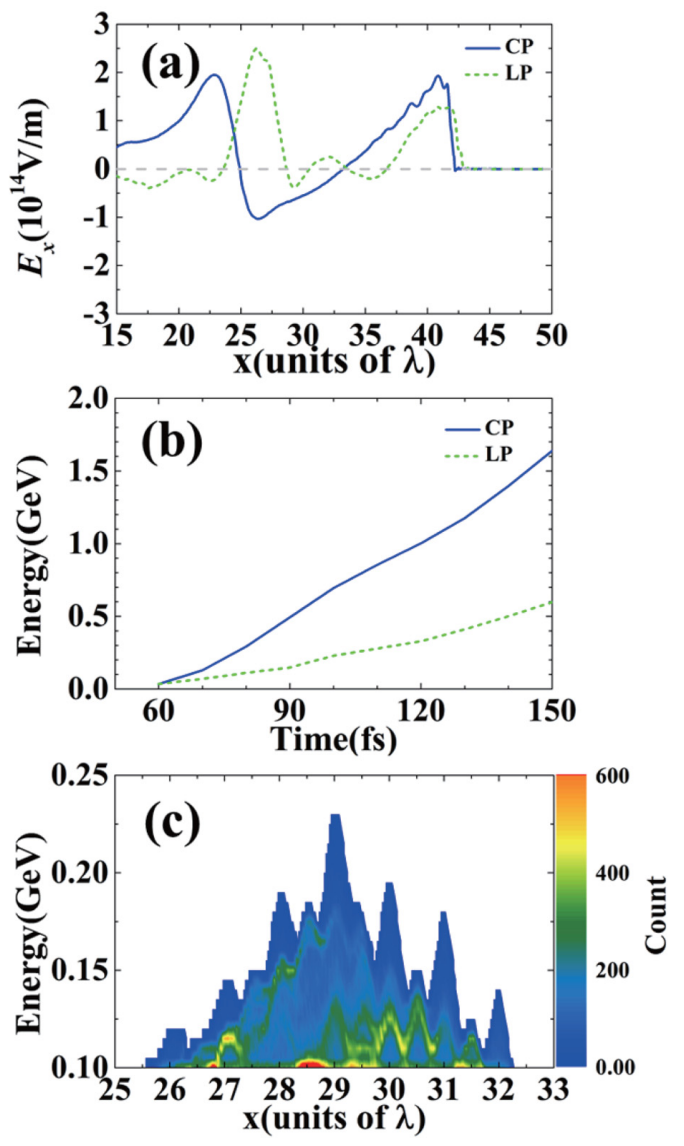

FIG. 4. (a) Distribution of $E_{x}$ with $x$ at 120 fs in two cases: a circularly polarized laser (blue solid line) and a linearly polarized laser (green dashed line). (b) History of maximum energy for protons. (c) Distribution of energy with $x$ at 100 fs for the case with a linearly polarized laser. The other simulation parameters are the same as those in Fig. 3(a).
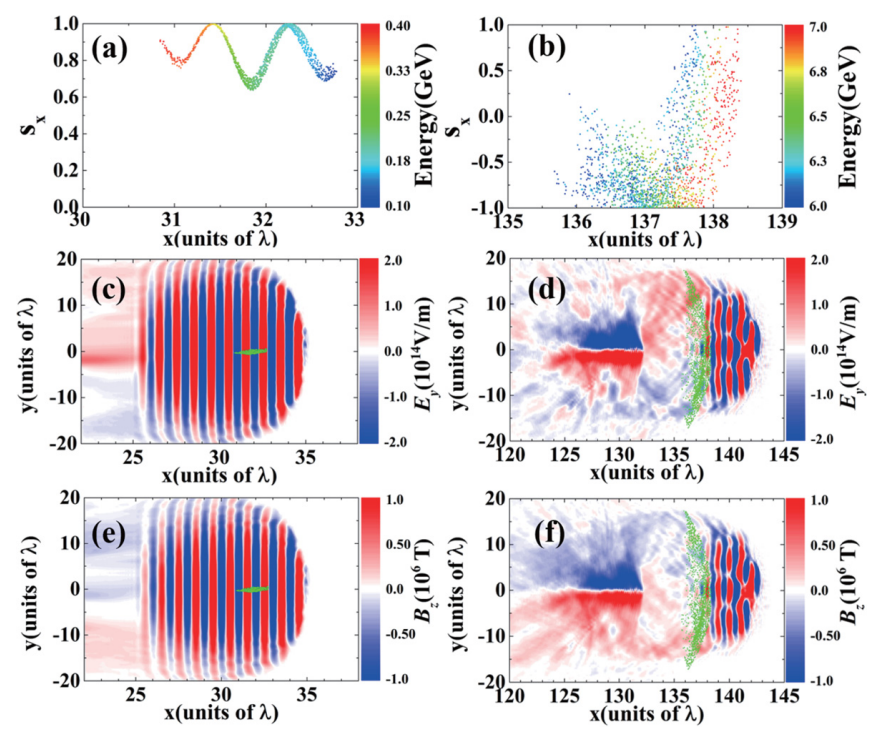

FIG. 5. Distribution of $s_{x}$ with $x$ for selected protons, as shown as Fig. 3(f), at different times: (a) $100 \mathrm{fs}$ and (b) $400 \mathrm{fs}$. Their positions are denoted by green dots in (c)-(f). Also shown are the distribution of $E_{y}$ at the respondence times (c) $100 \mathrm{fs}$ and (d) $400 \mathrm{fs}$ and the distribution of $B_{z}$ at the respondence times (e) $100 \mathrm{fs}$ and (f) $400 \mathrm{fs}$.
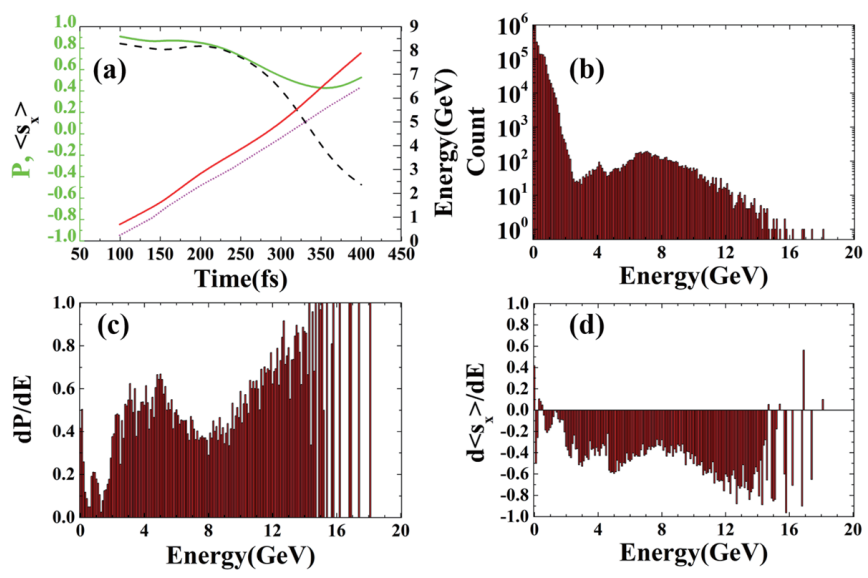

FIG. 6. (a) Polarization $P$ (green solid line) and $\left\langle s_{x}\right\rangle$ (black dashed line) of selected particles together with the average (purple dotted line) and maximum energies (red solid line), (b) energy spectrum at $800 \mathrm{fs}$, (c) distribution of $P$ with energy, and (d) distribution of $\left\langle s_{x}\right\rangle$ with energy.

The polarization of particle beams is defined as $P=$ $\sqrt{\left\langle s_{x}\right\rangle^{2}+\left\langle s_{y}\right\rangle^{2}+\left\langle s_{z}\right\rangle^{2}}$, where $\left\langle s_{i}\right\rangle$ is the average value in each direction. For the beam comprising the previously selected particles, this parameter is shown in Fig. 6(a). The maximum and average energies are also displayed here as the red solid and purple dotted lines, respectively. The maximum energy is nearly $8 \mathrm{GeV}$ and the average energy is $6.5 \mathrm{GeV}$ at $400 \mathrm{fs}$. Over time, the protons are accelerated while the value of $\left\langle s_{x}\right\rangle$ decreases, as denoted by the black dashed line. It should be noted that the polarization $P \simeq\left|\left\langle s_{x}\right\rangle\right|$ increases after $300 \mathrm{fs}$ according to this definition.

After protons become trapped in the wakefield, they are accelerated continuously, yielding a final energy spectrum of all protons at $800 \mathrm{fs}$ shown in Fig. 6(b). The corresponding distributions of $P$ and $\left\langle s_{x}\right\rangle$ are given in Figs. 6(c) and 6(d), respectively. Although the maximum energy is nearly $18.12 \mathrm{GeV}$, this is recorded by a single simulation particle, which means that $\left\langle s_{i}\right\rangle=s_{i}$ and its polarization $P$ is always 1, as shown in Fig. 6(c). In order to study the polarization with meaningful statistics, it is necessary to use $\left\langle s_{x}\right\rangle$ instead of $P$, where $\left\langle s_{y}\right\rangle$ and $\left\langle s_{z}\right\rangle$ are nearly zero owing to the azimuthal symmetry of the wake magnetic field. As revealed in Fig. 6(d), the $\left\langle s_{x}\right\rangle$ becomes negative, which causes the protons' polarization to change rapidly via this acceleration mechanism.

The number of protons with $E_{k}>4 \mathrm{GeV}$ is nearly $0.22 \mathrm{nC}$. Here $E_{k}>4 \mathrm{GeV}$ is used to isolate those protons which have undergone acceleration in the wakefield. In this case, the average energy is $7.65 \mathrm{GeV}$, the polarization is $P=0.44$, and the value of $\left\langle s_{x}\right\rangle$ is -0.42 . This result means that the depolarization of proton beams cannot be ignored in the present acceleration scheme, especially for the prepolarized uniform dense plasma. In addition, the polarization of protons may be conserved much better by overcoming the transverse defocusing effect.

\section{Effects of radiation reaction and mass ratio}

Although the radiation reaction (RR) effect for electrons is expected to become important for $a_{0} \geqslant 100$ [35], further 

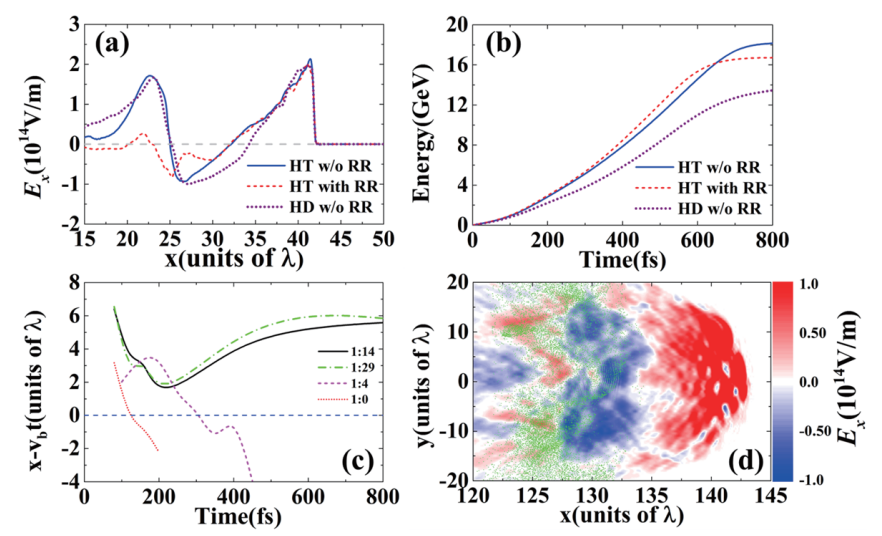

FIG. 7. (a) Longitudinal electric field at 120 fs and (b) history of the maximum proton energy for three cases: hydrogen-tritium plasma without the radiation reaction effect (blue solid line), hydrogen-tritium plasma with the RR effect (red dashed line), and hydrogen-deuterium plasma without the RR effect (purple dotted line). The electron density and other laser parameters are the same as those in Fig. 3(a). (c) History of the distance from protons with maximum $E_{k}$ to the position with $E_{x}=0$ in cases with different ratios. (d) Distribution of $E_{x}$ at 400 fs for the case with the ratio 1:4. The protons with $E_{k}>3 \mathrm{GeV}$ are shown as green dots.

simulations including QED effects show that both proton acceleration and polarization processes do not change significantly in our case, and the results presented here do not include generally QED effects. To verify this assumption, Fig. 7(a) shows a comparison with and without RR, and it is found that the effect is indeed relatively modest. Even with RR included, protons gain similar energies at the initial stage (before $400 \mathrm{fs}$ ), as shown in Fig. 7(b). Once protons have been captured by the wakefield, they go on to gain slightly more energy with RR included than without.

In this work, the heavier ions of a gas target are chosen as tritium, as in the work of Shen et al. [22], where the charge-to-mass ratio is $Z / A=1 / 3$. When the heavier ions are changed to deuterium, the $Z / A$ is $1 / 2$, similar to the average charge-to-mass ratio for most fully ionized gases. Figure 7(a) plots the distribution of $E_{x}$ at 120 fs for these charge-to-mass ratios, showing that the bubble radius, defined as the distance from the head of the bubble to its center $\left(E_{x}=0\right)$, is larger for smaller $Z / A$. The time history of maximum kinetic energy in Fig. 7(b) suggests the protons can be accelerated to higher energy in the case of tritium.

\section{E. Dependence on relative proton concentration}

The proton dynamics can be easily influenced by the relative proportion of hydrogen to tritium, as shown in Fig. 7(c). Here the position of $E_{x}=0$ is denoted by a blue dashed line. The proton positions with maximum $E_{k}$ for different ratios are shown by different colored lines. A position below zero means that the protons slip out from the acceleration region and can no longer be accelerated by the wakefield. This phenomenon occurs for the proton fraction above $20 \%$, a case illustrated in Fig. 7(d), where the protons with energy higher than $3 \mathrm{GeV}$ at $400 \mathrm{fs}$ are located in the $E_{x}<0$ region of the wakefield and thus they cannot be accelerated further.
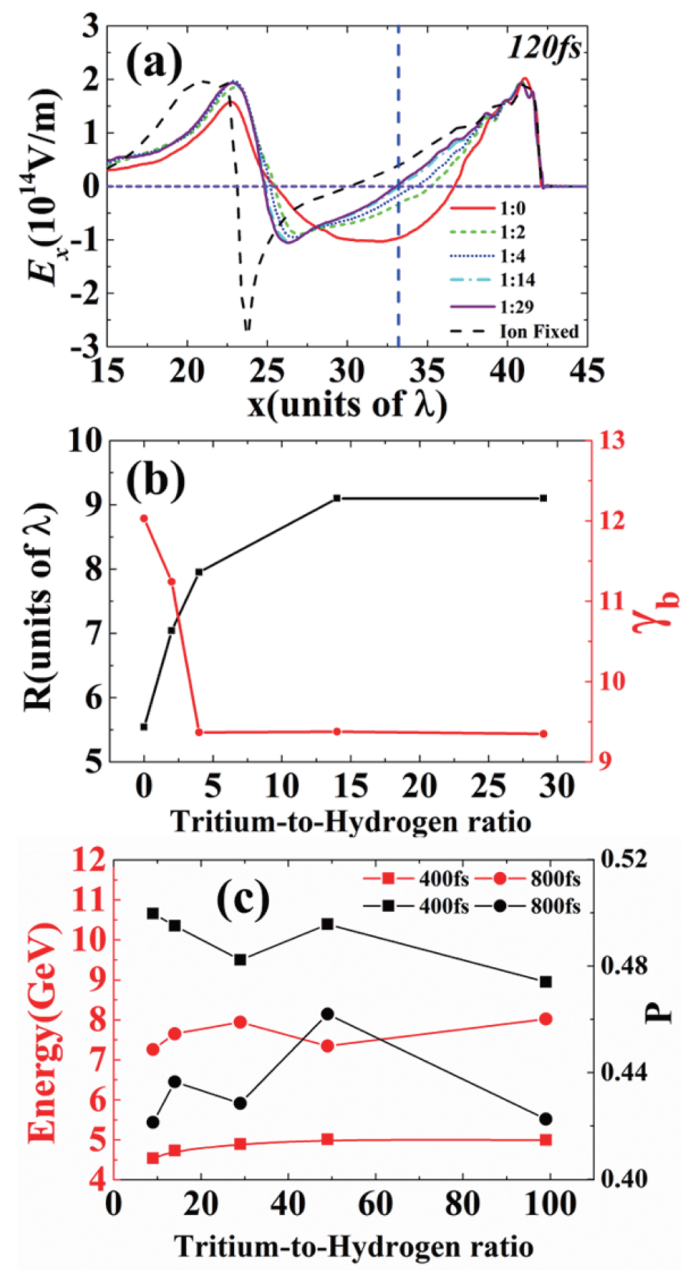

FIG. 8. (a) Distribution of $E_{x}$ with $x$ for different ratios. (b) Longitudinal radius of $E_{x}$ and the velocity of bubble, where $\gamma_{b}=$ $1 / \sqrt{1-\left(v_{b} / c\right)^{2}}$. (c) Average energy of protons with kinetic energy higher than $4 \mathrm{GeV}$ and their polarization $P$.

The $E_{x}(x)$ profiles at $120 \mathrm{fs}$ are shown in Fig. 8(a) for different ion ratios. In the case of electron acceleration in the bubble regime, the ion or proton is always considered immobile, as denoted by the black dashed line. For near-critical densities, the ion motion cannot be ignored. Here we can see that the $E_{x}$ profile is clearly altered by the ion composition, especially its slope $\Delta E_{x} / \Delta x$. With an increasing proportion of tritium, the distribution of $E_{x}$ approaches the fixed ion case. The bubble radius is defined as the distance from the head of the bubble to its center, where $E_{x}=0$ (blue dashed line). This parameter increases with the amount of tritium, whereas the velocity of the bubble decreases, as shown in Fig. 8(b). After being preaccelerated directly by the laser at the head of the bubble, it takes longer for protons to slip back to the center of the bubble with a larger radius, where the proportion of protons is smaller. This helps the proton obtain enough energy to catch up to the wakefield. Although our study has been restricted to hydrogen-tritium mixtures, our results should be equally applicable to $\mathrm{HCl}$ gas targets, where the density ratio of the proton and electron is $1: 18$. The density ratio of the proton and electron is more convenient, considering the 
species of ion has been changed and the motion of heavier ions, either tritium or chlorine, can be ignored.

Finally, we have studied the dependence of the average energy and polarization on the ion ratio [Fig. 8(c)]. Although the average $E_{k}$ of protons with $E_{k}>4 \mathrm{GeV}$ increases with a lower proton fraction, their polarization decreases. This difference in polarization can be accounted for by transverse defocusing of protons in the bubble regime, which on average could be exposed to a more intense magnetic field.

\section{SUMMARY}

We have studied the generation of high-energy proton beams including polarization properties in the interaction of ultrahigh-intensity lasers with near-critical-density plasmas. After preacceleration by a circularly polarized laser, the protons are trapped in the front region of a wakefield bubble and are further accelerated by the wakefield, where the acceleration gradient is nearly $10^{14} \mathrm{~V} / \mathrm{m}$. The protons can gain $10 \mathrm{GeV}$ in this field within $100 \mu \mathrm{m}$. Because the laser electric field is nearly $10^{14} \mathrm{~V} / \mathrm{m}$, the proton polarization can be affected by the laser. As the transverse size of the proton bunch increases, it experiences the full bubble magnetic field up to $0.1 \times 10^{6} \mathrm{~T}$, so its polarization is also modified by the wakefield. Finally, the relative proportions of hydrogen and tritium in the gas have a strong influence on the proton acceleration in this regime. The radius and the velocity of the accelerating field structure depend critically on the ion ratio. For sufficiently large ratios, a polarized proton beam can be trapped by the wakefield and accelerated to multi-GeV energies.

\section{ACKNOWLEDGMENTS}

Simulations were carried out on the JURECA supercomputer at Jülich Supercomputing Centre, which is granted from the Projects JZAM04 and LAPIPE. The work of A.H. and M.B. was carried out in the framework of the Jülich ShortPulse Particle and Radiation Center [36] and was supported by the Accelerator Technology Helmholtz Infrastructure consortium. This work was supported by the China and Germany Postdoctoral Exchange Program from the Office of China Postdoctoral Council and the Helmholtz Centre (Grant No. 20191016) and China Postdoctoral Science Foundation (Grant No. 2018M641993). This work was also supported by the Strategic Priority Research Program of Chinese Academy of Sciences (Grant No. XDA25050100), the National Natural Science Foundation of China (Grants No. 11975154, No. 11675108, No. 11655002, No. 11991074, and No. 11775144), and the Science Challenge Project (No. TZ2018005).
[1] D. Strickland and G. Mourou, Opt. Commun. 56, 219 (1985).

[2] E. Esarey, C. B. Schroeder, and W. P. Leemans, Rev. Mod. Phys. 81, 1229 (2009).

[3] A. Macchi, M. Borghesi, and M. Passoni, Rev. Mod. Phys. 85, 751 (2013).

[4] T. Tajima and J. M. Dawson, Phys. Rev. Lett. 43, 267 (1979).

[5] A. J. Gonsalves, K. Nakamura, J. Daniels, C. Benedetti, C. Pieronek, T. C. H. de Raadt, S. Steinke, J. H. Bin, S. S. Bulanov, J. van Tilborg, C. G. R. Geddes, C. B. Schroeder, C. Tóth, E. Esarey, K. Swanson, L. Fan-Chiang, G. Bagdasarov, N. Bobrova, V. Gasilov, G. Korn et al., Phys. Rev. Lett. 122, 084801 (2019).

[6] F. Wagner, O. Deppert, C. Brabetz, P. Fiala, A. Kleinschmidt, P. Poth, V. A. Schanz, A. Tebartz, B. Zielbauer, M. Roth, T. Stöhlker, and V. Bagnoud, Phys. Rev. Lett. 116, 205002 (2016).

[7] A. Higginson, R. Gray, M. King, R. Dance, S. Williamson, N. Butler, R. Wilson, R. Capdessus, C. Armstrong, J. Green et al., Nat. Commun. 9, 724 (2018).

[8] M. C. Downer, R. Zgadzaj, A. Debus, U. Schramm, and M. C. Kaluza, Rev. Mod. Phys. 90, 035002 (2018).

[9] M. Büscher, A. Hützen, L. Ji, and A. Lehrach, High Power Laser Sci. Eng. 8, e36 (2020).

[10] P. L. Anthony, R. G. Arnold, H. R. Band, H. Borel, P. E. Bosted, V. Breton, G. D. Cates, T. E. Chupp, F. S. Dietrich, J. Dunne, R. Erbacher, J. Fellbaum, H. Fonvieille, R. Gearhart, R. Holmes, E. W. Hughes, J. R. Johnson, D. Kawall, C. Keppel, S. E. Kuhn et al., Phys. Rev. Lett. 71, 959 (1993).

[11] H. Olsen and L. Maximon, Phys. Rev. 114, 887 (1959).

[12] P. J. Schultz and K. G. Lynn, Rev. Mod. Phys. 60, 701 (1988).
[13] Y.-F. Li, R. Shaisultanov, K. Z. Hatsagortsyan, F. Wan, C. H. Keitel, and J.-X. Li, Phys. Rev. Lett. 122, 154801 (2019).

[14] Y.-Y. Chen, P.-L. He, R. Shaisultanov, K. Z. Hatsagortsyan, and C. H. Keitel, Phys. Rev. Lett. 123, 174801 (2019).

[15] D. Sofikitis, C. S. Kannis, G. K. Boulogiannis, and T. P. Rakitzis, Phys. Rev. Lett. 121, 083001 (2018).

[16] M. Wen, M. Tamburini, and C. H. Keitel, Phys. Rev. Lett. 122, 214801 (2019).

[17] Y. Wu, L. Ji, X. Geng, Q. Yu, N. Wang, B. Feng, Z. Guo, W. Wang, C. Qin, X. Yan et al., New J. Phys. 21, 073052 (2019).

[18] N. Raab, M. Büscher, M. Cerchez, R. Engels, I. Engin, P. Gibbon, P. Greven, A. Holler, A. Karmakar, A. Lehrach et al., Phys. Plasmas 21, 023104 (2014).

[19] A. Hützen, J. Thomas, J. Böker, R. Engels, R. Gebel, A. Lehrach, A. Pukhov, T. P. Rakitzis, D. Sofikitis, and M. Büscher, High Power Laser Sci. Eng. 7, e16 (2019).

[20] M. Büscher, A. Hützen, I. Engin, J. Thomas, A. Pukhov, J. Böoker, R. Gebel, A. Lehrach, R. Engels, T. P. Rakitzis, and D. Sofikitis, Int. J. Mod. Phys. A 34, 1942028 (2019).

[21] L. Jin, M. Wen, X. Zhang, A. Hützen, J. Thomas, M. Büscher, and B. Shen, Phys. Rev. E 102, 011201(R) (2020).

[22] B. Shen, Y. Li, M. Y. Yu, and J. Cary, Phys. Rev. E 76, 055402(R) (2007).

[23] M. Liu, S. Weng, H. Wang, M. Chen, Q. Zhao, Z. Sheng, M. He, Y. Li, and J. Zhang, Phys. Plasmas 25, 063103 (2018).

[24] T. D. Arber, K. Bennett, C. S. Brady, A. Lawrence-Douglas, M. G. Ramsay, N. J. Sircombe, P. Gillies, R. G. Evans, H. Schmitz, A. R. Bell, and C. P. Ridgers, Plasma Phys. Control. Fusion 57, 113001 (2015). 
[25] S. Mane, Y. M. Shatunov, and K. Yokoya, Rep. Prog. Phys. 68, 1997 (2005).

[26] L. H. Thomas, Nature (London) 117, 514 (1926).

[27] R. W. Hockney and J. W. Eastwood, Computer Simulation Using Particles (CRC Press, Boca Raton, 1988).

[28] C. K. Birdsall and A. B. Langdon, Plasma Physics via Computer Simulation (CRC Press, Boca Raton, 2004).

[29] J. Thomas, A. Hützen, A. Lehrach, A. Pukhov, L. Ji, Y. Wu, X. Geng, and M. Büscher, Phys. Rev. Accel. Beams 23, 064401 (2020).

[30] N. Lemos, T. Grismayer, L. Cardoso, J. Geada, G. Figueira, and J. M. Dias, Phys. Plasmas 20, 103109 (2013).

[31] Y. Ma, D. Seipt, A. E. Hussein, S. Hakimi, N. F. Beier, S. B. Hansen, J. Hinojosa, A. Maksimchuk, J. Nees, K. Krushelnick,
A. G. R. Thomas, and F. Dollar, Phys. Rev. Lett. 124, 114801 (2020).

[32] W. L. Kruer and K. Estabrook, Phys. Fluids 28, 430 (1985).

[33] D. Del Sorbo, D. Seipt, T. G. Blackburn, A. G. R. Thomas, C. D. Murphy, J. G. Kirk, and C. P. Ridgers, Phys. Rev. A 96, 043407 (2017).

[34] D. Seipt, D. Del Sorbo, C. P. Ridgers, and A. G. R. Thomas, Phys. Rev. A 98, 023417 (2018).

[35] A. Di Piazza, C. Müller, K. Z. Hatsagortsyan, and C. H. Keitel, Rev. Mod. Phys. 84, 1177 (2012).

[36] M. Büscher, R. Adam, C. Tusche, A. Hützen, C. Wiemann, Y.-J. Chen, and C. M. Schneider, J. Large-Scale Res. Fac. 6, A138 (2020). 